\title{
CS duration and reinforcement schedule effects on conditioned enhancement and positive conditioned suppression
}

\author{
DONALD MELTZER \\ Southern Illinois University at Carbondale, Carbondale, Illinois
}

\begin{abstract}
Two groups of rats learned a discrimination in which responses were reinforced on a variableinterval (VI) schedule during one stimulus and extinguished during another. Eight conditioned stimuli (CSs), each followed by a food unconditioned stimulus (US), were presented during each session: four during the VI component and four during the extinction component. CS duration was $2 \mathrm{~min}$ for Group L and $30 \mathrm{sec}$ for Group S. Subjects in both groups responded at higher rates during CSs presented in the extinction component. Group L subjects also responded at higher rates during VI-component CSs, but Group S subjects responded at lower rates during VIcomponent CSs. These data indicate that reinforcement schedules may affect rate changes during CSs only within a limited range of CS durations.
\end{abstract}

A number of papers during the past few years have examined the interaction of operant and classical conditioning. In particular, there has been an interest in the effect of classically conditioned stimuli (CSs) an operant responses when both the reinforcement and the unconditioned stimuli (USs) are positive, or appetitive, stimuli. Several theorists have suggested that the use of positive reinforcement and USs would increase the strength of frequency of operant responses during the CS, an effect known as conditioned enhancement (e.g., Bindra, 1976; Rescorla \& Solomon, 1967; Trapold \& Overmier, 1972). However, a number of experiments have shown that animals may reduce their response rates during the $\mathrm{CS}$, an effect known as positive conditioned suppression. For example, some rats (Azrin \& Hale, 1969; Hake \& Powell, 1970; Van Dyne, 1971) and monkeys (Kelly, 1973; Miczek \& Grossman, 1971) have reduced their response rates during a CS under certain conditions, whereas subjects of the same species have increased their response rates under other conditions (Henton \& Brady, 1970; Meltzer \& Hamm, 1974, 1978).

Several variables seem to have affected the results of different experiments. Rats responded at higher than baseline rates during long CSs, and at lower than baseline rates during short CSs (Meltzer \& Brahlek, 1970; Meltzer \& Hamm, 1978), as did monkeys (Henton \& Brady, 1970). A localized CS, such as a lamp, produced much stronger response suppression in rats when it was far from the operant manipulandum than when it was close (Karpicke, 1978; Karpicke, Christoph, Peterson, \& Hearst, 1977).

This research was supported by a grant from the Office of Research Development and Administration, Southern Illinois University at Carbondale.

The author's address is: Department of Psychology, Southern Illinois University at Carbondale, Carbondale, IL 62901.
In addition, a number of experimenters have examined the role of the baseline schedule of reinforcement for operant responses. One of the first of these was Kelly (1973), who conditioned 2 rhesus monkeys to respond on both random-ratio and differential-reinforcement-of-low-rate (DRL) schedules. The monkeys were then presented with a 1-min light-CS/food-US pairing. Their rates increased during the CS if the baseline schedule was DRL, but decreased if the baseline schedule was random ratio. Unfortunately, different responses were required on the two schedules, and it was impossible to determine whether the schedules or the responses caused the difference in results. Meltzer and Hamm (1974) varied the reinforcement schedule in successive blocks of sessions while rats leverpressed for food reinforcement. A sucrose solution US followed a 2-min light CS in every session. There was a slight enhancement of responding with a variableinterval (VI) 1-min schedule, greater enhancement with a VI 4-min schedule, and much greater enhancement when operant responses were no longer reinforced. Although the results were clear, the changes in rate during the CS occurred on different days and may have been caused by the fact that the subjects were altering their rates as the schedule changed.

More recently, Lovibond (1981) performed an experiment in which rats were divided into three groups. Subjects in the first group were given repeated off-baseline pairings of a 10-sec auditory CS and a food US. For the second group, the CS was followed by a trace interval of 10 to $90 \mathrm{sec}$ before presentation of the US. For the third (control) group, the CS and US were unpaired. The rats were then conditioned to discriminate between one stimulus, during which leverpresses were reinforced on a variable-ratio (VR) 15 schedule, and another stimulus, during which responses were extinguished. After several sessions of discrimination training, a single CS, which 
was not followed by a US, was presented during each session. When the CS was presented during the VR 15 component, the rats in all three groups responded at reduced rats. When the CS was presented during the extinction component, rates decreased during the CS for the two experimental groups but were unchanged for subjects in the random control group. Edgar, Hall, and Pearce (1981) studied the effect of baseline schedules in an experiment in which three groups of rats were reinforced for responses on VI 30-sec, VI 60-sec, and VI 300-sec schedules, respectively. There were four 90 -sec noise CSs followed by four food pellets in each test session. Subjects in the VI 300-sec group increased their response rates during the CS, but rates in the other two groups were unaffected. The same experiment showed that CS rate increases could be produced with visual, as well as auditory, stimuli, even though the initial response to a light CS was rate reduction. Most recently, Ghezzi and Cheney (1983) reported mixed results from a study in which 2 rats learned an auditory discrimination. A fixed-ratio (FR) schedule was in effect during the tone, and a fixed-interval (FI) schedule in the absence of tone. A 10-sec light CS was presented either early or late in either the FR or FI schedule, but the only effect common to both subjects was an increase in rate during a CS presented in the early portion of a fixed interval.

The experiments described above support the hypothesis that rate is more likely to increase during a CS when baseline response rate and reinforcement frequency are low. However, there are questions about the generality of such an effect. For example, there has been no demonstration that a CS could cause both increases and decreases in the rate of the same response, depending upon the schedule of reinforcement used. The present study was an attempt to produce both increases and decreases in the rates of individual subjects in a single session.

\section{METHOD}

\section{Subjects}

The subjects were 24 male hooded rats between 90 and 120 days old at the beginning of the experiment. The animals were maintained on a 23-h food deprivation schedule throughout the experiment; they were fed immediately after a session. Water was always available in the animals' home cages.

\begin{abstract}
Apparatus
Six standard operant conditioning chambers were used. Each was equipped with a lever, a pellet dispenser, a liquid dipper, a cuelight, and a sonalert audio signal generator. The dry food used consisted of 45-mg Noyes pellets. A complete description of the chambers was given by Meltzer and Hamm (1974). White noise was present in the experimental room during all sessions. Programming and recording equipment were in an adjacent room.
\end{abstract}

\section{Procedure}

The subjects were shaped to eat from the food hopper, drink from the dipper, and press the lever during the first two sessions. They were then reinforced on a VI 1-min schedule for pressing the lever during the next two sessions. From the fifth session until the end of the experiment ( 36 sessions), responses were reinforced on a VI 1-min schedule during 25-min of the 47-min session and were never reinforced during the remaining $22 \mathrm{~min}$ of the session. For half the subjects, the cuelight was on while the VI schedule was in effect and off while the extinction schedule was in effect; for the remaining subjects, the cue light was on during the extinction component and off during the VI component. The VI 1-min schedule was always in effect during the first $3 \mathrm{~min}$, and extinction and reinforcement components alternated for the remainder of the session. The remaining $22 \mathrm{~min}$ of the VI schedule were divided into four VI components of different durations: 4, 5, 6, and $7 \mathrm{~min}$. The 22 min of extinction were divided in the same way.

After 16 sessions of discriminative conditioning, a tone CS was introduced, followed by a food pellet US. The CS-US interval was $2 \mathrm{~min}$ for half the subjects (Group L), and $30 \mathrm{sec}$ for the remaining subjects (Group S). The tone was on during the entire CS-US interval.

There were eight CS-US trials in each session, four during the VI component and four during the extinction component. No CS ever began during the first $90 \mathrm{sec}$ of a component. However, a CS-US interval could begin at any time during the remainder of the component, as long as there was enough time left in the component for the trial to be completed. Responses during the CS were recorded separately during the VI and extinction components and were compared with baseline response rates during the same components. Because the CS durations were different, the method of computing baseline rates differed slightly for the two groups. For Group $S$, the baseline rate was the response rate during the $30 \mathrm{sec}$ preceding the CS. For Group L, a similar procedure would have required that the trials not begin until at least 2 min into the component, and then sometimes would have required that the baseline rate include responses during the first minute of the component. Therefore the extinction baseline rate for Group $\mathrm{L}$ was measured by counting all responses during extinction components except for responses during the $\mathrm{CS}$ and during the first minute of the component. The same procedure was used to count the VI baseline responses during the VI components.

This procedure was followed for the next 20 sessions. Sessions were scheduled 5 days a week throughout the experiment.

\section{RESULTS}

Data from the last 20 sessions of the experiment were anlayzed. Mean pre-CS and CS response rates were computed for each session and these data were combined in mean values for five successive four-session blocks. Baseline response rates were compared to response rates during the CS for subjects in both groups. Figure 1 shows the mean baseline rates and CS rates of Group L subjects during the VI and extinction components of each block of sessions. Mean rates were significantly higher during the VI component $[F(1,5)=21.22, p<.01]$ across all the session blocks, but response rate increased during the VI component over session blocks while remaining fairly stable during the extinction component. This interaction was significant $[F(4,20)=7.77, p<.01]$, and tests of simple main effects confirmed the significant increase of VI rates. Tests of simple main effects also showed that the difference between VI and extinction rates was significant during each block of sessions.

Figure 1 also shows that CS rates were lower than baseline rates during the first session block and were higher than baseline rates during the later blocks. This interaction was also significant $[F(4,20)=7.35, p<.01]$. Tests of simple main effects showed that the difference between baseline and CS rates was not significant during the first three blocks but that CS rate was significantly higher during the last two blocks of sessions.

Figure 2 shows the performance of subjects in Group $S$ over the five blocks of sessions. Response rates were significantly higher during the VI components than during 
the extinction components $[F(1,5)=16.37, p<.01]$. However, subjects in this group did not respond in the same way to CS presentations during the VI and extinction components. Mean response rate decreased during the CS, as compared to baseline rate, in the VI component. Mean response rate increased during the CS, as compared to baseline rate, in the extinction component. The interaction was significant $[F(1,5)=84.46, p<.01]$, and tests of simple main effects showed that the difference between baseline and CS rates was significant on both reinforcement schedules.

A third analysis compared baseline and CS rates of the two groups during the VI components of the five blocks of sessions. A comparison of Figures 1 and 2 shows that the baseline rates of subjects in the two groups were similar, but that Group L subjects responded at higher rates during the CS than did Group S subjects. The interaction was significant $[F(1,10)=9.77, p<.01]$, and tests of simple main effects confirmed that the difference between baseline rates was not significant, whereas the difference between CS rates was significant.

The final analysis compared baseline and CS rates of the two groups of subjects during the extinction components of the sessions. There were no differences between

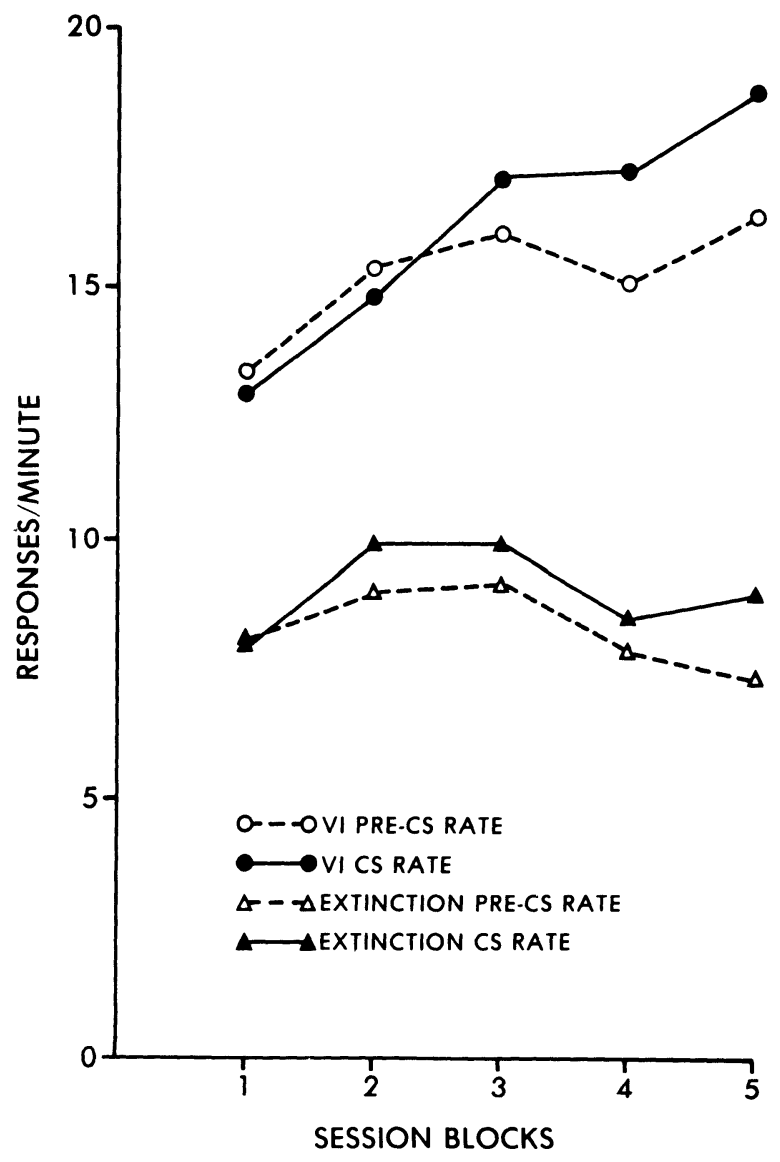

Figure 1. Mean pre-CS and CS response rates for subjects in Group L. Rates were recorded separately during VI and extinction components.

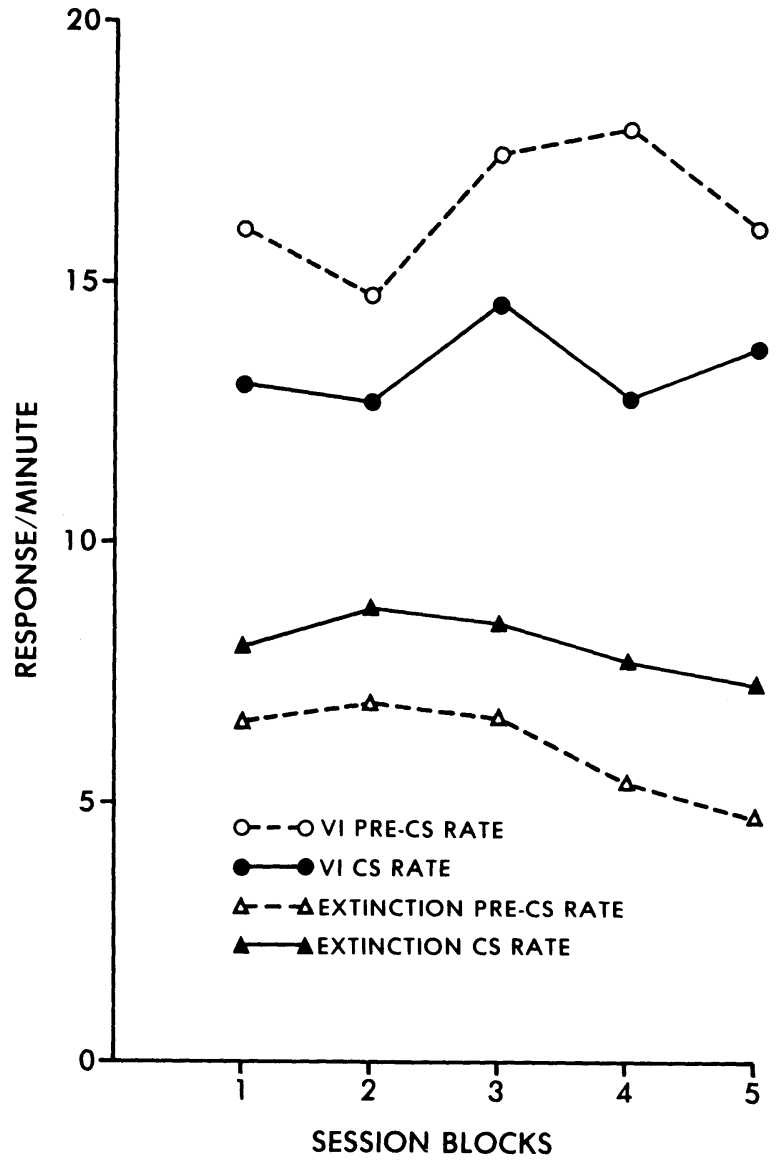

Figure 2. Mean pre-CS and CS response rates for subjects in Group S. Rates were recorded separately during VI and extinction components.

the rates of subjects in the two groups in either stimulus condition. The only significant result was that rates in both groups were higher during the $\operatorname{CS}[F(1,10)=13.41, p<$ $.01]$.

\section{DISCUSSION}

The results of this experiment show that the schedule of reinforcement was a factor in determining direction of change in subjects' response rates when short, but not long, CS-US intervals were used. They are consistent with the results reported by Meltzer and Hamm (1974), who found that rate increases during a pre-food CS were inversely related to reinforcement frequency or baseline response rate. However, in that study such effects were found when a 2-min CS was used, whereas in the present study no such effects were found during 2-min CSs. The likeliest explanation is that Meltzer and Hamm were testing different schedules in successive blocks of sessions and their results were affected by the transitions. The present results are also consistent with those of Kelly (1973), and show that the effects he reported were not dependent upon the use of primate subjects or different responses during long and short CSs. Edgar, Hall, and Pearce (1981) also found that rates increased during the CS when reinforcement frequency and response rates were low, but Lovibond (1981) did not find any effect of reinforcement on rates during the $\mathrm{CS}$. These experiments indicate that CS duration may be a major variable in determining whether schedules will affect rate. Lovibond used a 10-sec CS; in the present experiment an effect was found with a 30-sec CS; Kelly (1973) found an effect with a 60-sec CS, as did Edgar, Hall, and Pearce (1981) with a 90-sec CS; Meltzer and 
Hamm (1974) found an effect with 2-min CSs during transitional states, but in the present study no effect was found with 2 -min CSs presented on a stable discrimination baseline. In short, it appears that baseline schedules may alter the direction of rate changes during a pre-food CS when CS duration is in the $30-90 \mathrm{sec}$ range, but have no effect when CS duration is shorter or longer.

A related question is whether rate changes actually occur during the CS or are a consequence of different baseline rates. In the present study, rates increased during the $\mathrm{CS}$, regardless of its duration, when it was presented during an extinction component. Thus, conditioned enhancement may be a result of low response rates or of nonreinforcement. In other words, the response rate during a CS presented in extinction could only increase, and the effect would be determined by baseline conditions. But the comparison of rates during the VI component showed a different result. There was no difference between the groups' baseline rates, but Group $S$ subjects responded at decreased rates during the CS, whereas Group L subjects responded at increased rates. Moreover, the difference between the CS rates of the two groups was significant. Therefore, the CS had an effect on rates which depended upon CS duration and could not be a consequence of differences in either baseline rates or reinforcement frequency.

\section{REFERENCES}

Azrin, N. H., \& Hake, D. F. (1969). Positive conditioned suppression: Conditioned suppression using positive reinforcers as the unconditioned stimuli. Journal of the Experimental Analysis of Behavior, 12, 167-173.

BINDRA, D. (1976). A theory of intelligent behavior. New York: Wiley. Edgar, D., Hall, G., \& Pearce, J. M. (1981). Enhancement of foodrewarded instrumental responding by an appetitive conditioned stimulus. Quarterly Journal of Experimental Psychology, 33B, 3-19.

GHEZZI, P. M., \& CHENEY, C. D. (1983). Stimulus and prefood stimulus effects on fixed-interval and fixed-ratio responding. Bulletin of the Psychonomic Society, 21, 491-494.

HAKE, D. F., \& Powell, J. (1970). Positive reinforcement and suppression from the same occurrence of the unconditioned stimulus in a positive conditioned suppression procedure. Journal of the Experimental Analysis of Behavior, 14, 247-257.

Henton, W. W., \& BRADY, J. V. (1970). Operant acceleration during a pre-reward stimulus. Journal of the Experimental Analysis of Behavior, 13, 205-209.

KARPICKE, J. (1978). Directed approach responses and positive conditioned suppression in the rat. Animal Learning and Behavior, 6, 216-224.

Karpicke, J., Christoph, G., Peterson, G., \& Hearst, E. (1977). Signal location and positive versus negative conditioned suppression in the rat. Journal of Experimental Psychology: Animal Behavior Processes, 3, 105-118.

KeLLY, D. D. (1973). Long-term prereward suppression in monkeys unaccompanied by cardiovascular conditioning. Journal of the Experimental Analysis of Behavior, 20, 93-104.

LoviBOND, P. F. (1981). Appetitive Pavlovian-instrumental interactions: Effects of inter-stimulus interval and baseline reinforcement conditions. Quarterly Journal of Experimental Psychology, 33B, 257-269.

Meltzer, D., \& BrahleK, J. A. (1970). Conditioned suppression and conditioned enhancement with the same positive UCS: An effect of CS duration. Journal of the Experimental Analysis of Behavior, 13, 67-73.

Meltzer, D. \& Hamm, R. J. (1974). Conditioned enhancement as a function of schedule of reinforcement. Bulletin of the Psychonomic Society, 3, 99-101.

Meltzer, D., \& Hamm, R. J. (1978). Differential conditioning of conditioned enhancement and positive conditioned suppression. Bulletin of the Psychonomic Society, 11, 29-32.

MiczeK, K. A., \& Grossman, S. P. (1971). Positive conditioned suppression: Effects of CS duration. Journal of the Experimental Analysis of Behavior, 15, 243-247.

Rescorla, R. A., \& Solomon, R. L. (1967). Two-process learning theory: Relationship between Pavlovian conditioning and instrumental learning. Psychological Review, 74, 151-182.

TRAPOLD, M. A., \& OVERMieR, J. B. (1972). The second learning process in instrumental learning. In A. J. Black \& W. F. Prokasy (Eds.), Classical conditioning: II. Current research and theory. New York: Appleton-Century-Crofts.

VAN DYNE, G. (1971). Conditioned suppression with a positive US in the rat. Journal of Comparative \& Physiological Psychology, 77, 131-135.

(Manuscript received for publication March 31, 1986.) 